\title{
Diversity enforces social exclusion: Does exclusion never cease?
}

\author{
Saqib Amin \\ National College of Business Administration \\ \& Economics
}

\begin{abstract}
Diversity plays a vital role in sustainable development of any country. Discrimination, segregation and bigotry are escalating the pressure on the world's population. This study aims to investigate the impact of ethnic and religious diversity on social exclusion (in the form of economic exclusion, exclusion from public service and exclusion from civic and public participation as indicated by United Nations Development Program (UNDP) by using data from 187-countries. Based on panel data methodology this study concludes that diversity, either ethnic or religious or both, can increase social exclusion and affect wellbeing at a population level. This study suggests that ethnic and religious diversity is an inherent part of most societies in a globalised world and is unlikely to be halted yet the unintended negative impacts of such increased diversity can be minimized by establishing cohesiveness in society.
\end{abstract}

Keywords: Ethnic Diversity; Religious Diversity; Social Exclusion, United Nation Development Program (UNDP).

\section{Introduction}

Diversity is a complex, multidimensional and worldwide phenomenon. It has been important for the prosperity of any country since globalization. Due to its multidimensionality, there are numerous definitions used to explain the concept of diversity. Ethnic heterogeneity and religious divisions have become burning global issues (Azam, 2001). It is truly hard to find any place or field where there is no existence of more than one ethnic or religious group. The flow of ethnic groups within totally different cultures and norms is increasing in volume every year (Barth, 1998; Bates, 2000; Castles, 2000; Sung, 2014). Cross-border communities and the coexistence of multiple communities in local spaces are common, not the exception. A more severe challenge relates to maintaining cohesiveness when people discriminate on the basis of emotional, cultural and religious identities. However, international migration, as well as persistent social changes and pluralism, are having an economic, social and political impacts around the world.

Corresponding author: Saqib Amin (saqib.amin000@gmail.com) 
Diversity plays a central role in societal transformation but higher diversity often limits equal opportunity to all the members of society (Thomas \& Ely, 2001) and can be the cause of social exclusion. Social exclusion is essentially social disadvantage and relegation to the fringe of society (Simandan, 2010). This terminology is well understood across disciplines such as psychology, sociology, politics, education and economics (Peace, 2001; Silver, 2007). The United Nations Development Program (UNDP) virtual round table defined exclusion as any distinction, restriction or preference, which is based on grounds such as race, colour, sex, language, religion, social class, and consequently nullifies or impairs the recognition, enjoyment or exercise by all persons, on an equal footing, of all rights and freedoms (Peace, 2001; UNDP, 2011), Diversity is one of the main components which causes societal alteration and which in turn forces individuals in the direction of exclusion or the margins of society. (Walsh, 2006; Young, 2002).

Levitas et al. (2007) showed that diversity affects the well-being of individual's, equity and cohesion of society (social inclusion) as a whole. The result can be an exclusionary process whereby people are cut off for a significant period of their lives from institutions and services, social networks and developmental opportunities that the great majority of society enjoys (Mathieson et al., 2008). Aasland and Fløtten (2001) argue that social exclusion is about more than income poverty and becomes more critical when people or areas face a combination of linked problems, such as discrimination, poor skills, low incomes, unemployment, poor housing, high crime and family breakdown (Amin \& Ahmad, 2018; Jehoel-Gijsbers \& Vrooman, 2007). These problems are linked and mutually reinforcing (Unit, 2004). These excluded individuals show inability to participate in the basic social, economic and political process of the society (Peleah \& Ivanov, 2013; Thorat \& Neuman, 2012) and are more likely to break the rules and fail to follow social norms of the society (Popay et al., 2008).

Rawal (2008) explore the extent or deepness of social exclusion by elaborating on the multifarious aspects of culture, institutions and social deprivation. The strength of social exclusion is derived through social deprivation/distinction to poverty, exclusion from intergroup class, norms status and political power (Benington \& Geddes, 2013). In this view, social exclusion theoretically emerges from insufficient access to social rights, material deprivation, limited social participation and a lack of normative integration. It is then regarded as the combined result of macro-societal changes including social, demographic, economic, labor market developments, social norms, government legislation and social policy (Vrooman \& Hoff, 2013).

Levitas (2004) pointed out that dealing with social exclusion as a single discourse for Europe would be unfair and inappropriate as it is spreading all over the world rather than only in advanced economies. The different patterns of exclusion increase day by day, which damages the prosperity of the country. Cultural factors, namely ethnic diversity has also received much attention in relation to building social cohesiveness. A strong but mixed relationship exists between diversity, social cohesion and institutional quality (Amin, 2019; Ellis, Beaver, \& Wright, 2009; Hooghe \& de Vroome, 2016), which is mostly based on geographical areas, such as the sphere of exclusion is different in heterogeneous communities as compared to homogenous communities (Ellis et al., 2009). In this modern era, where societal development is putting people towards the centre of development processes, social inclusion is found to be a critical component for economic development and sustainability of a country (DESA, 2007). On the other hand, diversity can create conflict and clashes in societal development. The aim of this study is to analyse diversity (ethnic or religious) and social exclusion from a worldwide perspective.

The rest of the paper is as follows: Section II consists of the theoretical framework. Section III contains methodology and data sources. The next section highlights empirical results and 
the last section concludes the study, providing brief policy recommendations based on the findings.

\section{Theoretical Framework}

Recent literature shows various determinants of social inclusion/exclusion and its relationship with wellbeing. The development process started by Sen's claims, that the capability approach is not a theory of justice or equality, but a theory of a society which directly links high levels of wellbeing and quality of life (Robeyns, 2005).

According to relational theory, to create a society in which there is equal respect, regardless of whether there is any actual material equality of condition, what matters is the relations between people in a diverse society (Rosen, 2004). The provision of sufficient capabilities in heterogeneous communities is required so that no-one is forced to feel ashamed of their circumstances. However, the theory of social exclusion is related to choice, welfare and fairness for each individual within society, and how goods are provided (Barry, 2002).

Many have suggested, that the capability approach is not a neutral formation of a person's good and hence cannot be a theory, at least not a liberal theory, or theory of justice (Schumpeter, 2017). In this context, capability approaches are considered the best way to conceptualize social exclusion, (Fleurbaey, 2002; Robeyns, 2005). So the concern with social exclusion can be seen by combined effects of capabilities theory of social justice ${ }^{1}$ and the theory of equality of opportunity (Miller, 2006). This approach reflects the multidimensionality of social exclusion (Levitas et al., 2007).

Interestingly, Wolff and De-Shalit (2007) and Esping-Andersen, Gallie, Hemerijck, and Myles (2002) have both endorsed the capability approach but not as a theory of quality of life or social welfare. They focus on providing a minimum level of functioning as a way of addressing disadvantage. They have argued that clusters of disadvantage are created, much like the set of mutually reinforcing linked problems that are the concern of analysts of social exclusion. These interlinked problems are the most critical for social action and present empirical questions for social welfare and decision-makers.

Furthermore, the theory of relational equality does insist upon a sufficient level of functioning, as to ensure respect among citizens (Vigoda, 2002). However, most individuals excluded from social opportunities relate or identify as a marginalised underclass (Lister, 1998). Such groups often experience 'exclusion' from mainstream society not only in an economic sense, but also as a cultural, political and organisational phenomenon (Karlsen \& Nazroo, 2002; McAdam, 2000). The focus on citizenship also links with one of the key problems experienced by theorists of social exclusion; the issue of inclusion and social diversity (Uslaner, 2012).

The question of how to combat social exclusion, poverty, and ethnic discrimination is discussed today in tandem with calls to increase economic efficiency, growth, and deregulation of the labour market (Silver \& Miller, 2003). Talk about diversity has become social and political rhetoric that conceals growing unregulated new forms of discriminatory ethnic divisions. By using the theoretical framework and empirical studies of Alesina et al. (2003), this study analyses the direct effect of religious and ethnic diversity on social exclusion, through adopting the standard model of Jehoel-Gijsbers and Vrooman (2007)

\footnotetext{
${ }^{1}$ Indeed, the SEU's 2004 report indicate social exclusion as the problem, but the solution is not "inclusion", but greater equality of opportunity. That is, the goal of policies to reduce social exclusion is to promote equality of opportunity (2004: 34$)$.
} 
such as;

$$
S E_{j, t}=a+\beta_{i}(d i v)_{j}+\beta_{i}\left(X^{\prime}\right)_{j}+\varepsilon_{j, t}
$$

Whereas, "SE" indicate indicators of social exclusion, "div" shows the diversity both ethnic and religious, " $X$ " shows the other control variables such as GDP per capita, urbanization, education and health expenditure, population density, literacy rate, institutional quality and "et" is the error term.

\section{Methodology and Data Sources}

In order to find the relationship between diversity and social exclusion, this study used panel data methodology to explore this nexus and dynamics for empirical analysis. Basically, panel data methodology is the mixture of cross-sectional and time series data which does not just increase the power and size of data but also restructuring or re-examining effects that are difficult to distinguish with only cross-sections or time series data (Hsiao, 1986). Baltagi and Kao (2001) describes the key advantages of using panel data, such as how heterogeneity in countries is absent when using aggregate time series data. Panel data permits more variability, less collinearity among variables, while the cross sections of time series provide more degree of freedom and more efficiency when estimating models.

Panel data under fixed effect can be expressed as;

$$
Y_{i t}=\alpha_{i}+X_{i t}^{\prime} \beta+v_{i t}
$$

Whereas $\mathrm{t}=1 \ldots \ldots \mathrm{T}, \mathrm{i}=1 \ldots \ldots \mathrm{N}$ and $v_{i t}$ is the error term. In a fixed effects model, the unobserved variables are permitted to have any relations whatever with the observed variables. This shows the rationality following the assumption of the relationship between error term and outcome variables. One of the important assumptions of the fixed effect model is that time-invariant characteristics are unique and should not be correlated with other characteristics of the individuals. So, this technique is more suitable to empirical testing the relationship between diversity and social exclusion.

Dataset of all the variables used in this paper are freely available/access for empirical testing. ${ }^{2}$ This paper initially looks at the relationship between diversity and social exclusion by using panel data analysis of 187-countries (listed in appendix), for the time period from 1990 to 2010 with time interval of averages of 05-years. Diversity is measured by using the following formula of Alesina et al. (2003) such as;

$$
\text { FRACT }_{j}=1-\sum_{\mathrm{i}=1}^{\mathrm{N}} \mathrm{D}_{\mathrm{ij}}^{2}
$$

Whereas, $\mathbf{D}_{\mathrm{ij}}$ is the share of group $\mathrm{i},(\mathrm{i}=1 \ldots \ldots \mathrm{N})$ in the country $\mathrm{j}$. The range of the result is between $0-1$. Zero " 0 " means a homogenous country and " 1 " shows total heterogeneity in a country. Social exclusion means excluded individuals from society on the basis of economic, political and civic participations. ${ }^{3}$ This study therefore followed the definition of UNDP (2013) for measurement of social exclusion (in form of exclusion from economic, public services and civic and public participations).

\footnotetext{
${ }^{2}$ For more details of variables description and data source, see the appendix at the end of this study.

${ }^{3}$ For more information about all the indicators of social exclusion, see the appendix at the end of this study.
} 


\section{Empirical Results}

Table 1a - Effects of ethnic and religious diversity on Economic Exclusion

\begin{tabular}{|c|c|c|c|c|c|c|c|c|c|c|}
\hline Dependant & Coff. & $\mathrm{C}$ & R-sqd. & Obs. & $\begin{array}{c}\text { Cros. } \\
\text { Sec }\end{array}$ & Coff. & $\mathrm{C}$ & R-sqd. & Obs. & $\begin{array}{c}\text { Cros. } \\
\text { Sec } \\
\end{array}$ \\
\hline Variables & (e) & (e) & (e) & (e) & (e) & (r) & (r) & (r) & (r) & (r) \\
\hline UNEMP & $\begin{array}{l}2.203^{\top \oplus} \\
(0.938)\end{array}$ & $\begin{array}{c}10.123^{\text {TФ }} \\
(0.49)\end{array}$ & 0.176 & 716 & 148 & $\begin{array}{l}2.587^{\mathrm{T}} \\
(1.086)\end{array}$ & $\begin{array}{l}10.27^{\mathrm{T \top}} \\
(0.492)\end{array}$ & 0.075 & 749 & 155 \\
\hline GINI & $\begin{array}{l}1.459^{\mathrm{T \top}} \\
(2.46)\end{array}$ & $\begin{array}{l}3.346^{\mathrm{T \top}} \\
(1.124)\end{array}$ & 0.132 & 233 & 100 & $\begin{array}{l}4.080^{\mp} \\
(2.91)\end{array}$ & $\begin{array}{l}40.39^{\mp \top \top} \\
(1.181)\end{array}$ & 0.017 & 250 & 105 \\
\hline $\begin{array}{l}\text { Depth Food Deficit } \\
\text { (kilocalories) }\end{array}$ & $\begin{array}{l}0.507^{\mathrm{TT}} \\
(3.460)\end{array}$ & $\begin{array}{l}1.036^{\mp \top} \\
(0.082)\end{array}$ & 0.24 & 201 & 79 & $\begin{array}{l}0.727^{\mp \top \Phi} \\
(0.715)\end{array}$ & $\begin{array}{l}0.272^{\mp \top} \\
(13.59)\end{array}$ & 0.027 & 194 & 80 \\
\hline $\begin{array}{l}\text { Poverty (Head count } \\
\text { Ratio) }\end{array}$ & $\begin{array}{l}0.612^{\top \oplus \Phi} \\
(0.007)\end{array}$ & $\begin{array}{l}0.301^{\text {ФФ }} \\
(0.934)\end{array}$ & 0.101 & 681 & 151 & $\begin{array}{c}3.295^{\mathrm{T \top}} \\
(0.51)\end{array}$ & $\begin{array}{l}0.284^{\top \top \top} \\
(0.436)\end{array}$ & 0.034 & 690 & 148 \\
\hline Poverty (Gap Ratio) & $\begin{array}{l}3.144^{\mathrm{T \top}} \\
(1.439)\end{array}$ & $\begin{array}{l}3.700^{\mathrm{TФ}} \\
(3.572)\end{array}$ & 0.259 & 575 & 141 & $\begin{array}{l}0.153^{\text {ТФ }} \\
(3.413)\end{array}$ & $\begin{array}{c}0.261^{\text {ФФ }} \\
(0.92)\end{array}$ & 0.171 & 545 & 139 \\
\hline Corruption Score & $\begin{array}{l}06.16^{\mathrm{TT}} \\
(0.302)\end{array}$ & $\begin{array}{l}5.453^{\text {ФФ }} \\
(1.804)\end{array}$ & 0.112 & 542 & 144 & $\begin{array}{l}10.75^{\text {tक }} \\
(4.256)\end{array}$ & $\begin{array}{c}37.68^{\mp \top \top} \\
(1.92)\end{array}$ & 0.011 & 573 & 152 \\
\hline $\begin{array}{l}\text { Intentional Homicides } \\
\text { (per } 100,000 \text { people) }\end{array}$ & $\begin{array}{l}6.588^{\mp \top \top} \\
(2.284)\end{array}$ & $\begin{array}{l}5.734^{\mathrm{T \top}} \\
(1.152)\end{array}$ & 0.229 & 532 & 148 & $\begin{array}{c}-0.21 \\
(2.573)\end{array}$ & $\begin{array}{l}8.414^{\mathrm{TT}} \\
(1.146)\end{array}$ & 0.120 & 560 & 155 \\
\hline $\begin{array}{l}\text { Out-of-pocket health } \\
\text { expenditure }\end{array}$ & $\begin{array}{c}0.057^{\mathrm{T}} \\
(0.0355)\end{array}$ & $\begin{array}{l}0.006^{\mathrm{T \top}} \\
(0.0002)\end{array}$ & 0.013 & 239 & 89 & 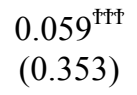 & $\begin{array}{l}0.18^{\mathrm{T \top}} \\
(0.002)\end{array}$ & 0.0835 & 194 & 78 \\
\hline
\end{tabular}

Note; Results shows the robust analysis using the ordinary least square. (e) and (r) columns show the values of independent variable i.e. ethnic and religious diversity respectively. Coff.. C, R-sqd., Obs, Cros.Sec shows the coefficient value, constant,

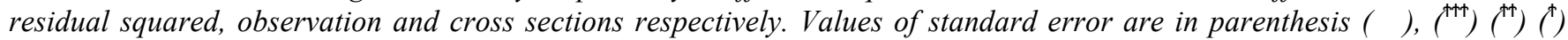
shows the level of significance at $1 \%, 5 \%$ and $10 \%$ respectively. 


\section{Table 1b - Effects of ethnic and religious diversity on exclusion from public services}

\begin{tabular}{|c|c|c|c|c|c|c|c|c|c|c|}
\hline Dependant & Coff. & $\mathrm{C}$ & R-sqd. & Obs. & Cros.Sec & Coff. & $\mathrm{C}$ & R-sqd. & Obs. & Cros.Sec \\
\hline $\begin{array}{l}\text { Out of school } \\
\text { Children }\end{array}$ & $\begin{array}{l}3.27^{\mathrm{T \top}} \\
(0.508)\end{array}$ & $\begin{array}{c}0.291^{\mathrm{T}} \\
(1.32)\end{array}$ & 0.133 & 432 & 129 & $\begin{array}{c}3.295^{\mathrm{T \top}} \\
(0.51)\end{array}$ & $\begin{array}{l}0.297^{\mathrm{T \top}} \\
(0.449)\end{array}$ & 0.359 & 411 & 127 \\
\hline $\begin{array}{l}\text { Lifetime risk of } \\
\text { maternal death }(\%)\end{array}$ & $\begin{array}{l}3.336^{\text {ТФ }} \\
(0.484)\end{array}$ & $\begin{array}{l}0.859^{\mathrm{T \top}} \\
(0.871)\end{array}$ & 0.086 & 390 & 101 & $\begin{array}{l}0.231^{\text {Фஈ }} \\
(0.034)\end{array}$ & $\begin{array}{l}0.173^{\text {ТФ }} \\
(0.002)\end{array}$ & 0.003 & 290 & 89 \\
\hline $\begin{array}{l}\text { Low-birthweight } \\
\text { babies (\% of births) }\end{array}$ & $\begin{array}{l}0.413^{\mathrm{T \top}} \\
(0.412)\end{array}$ & $\begin{array}{l}0.371^{\mathrm{T \top}} \\
(1.043)\end{array}$ & 0.064 & 289 & 78 & $\begin{array}{c}0.280^{\mathrm{T \top}} \\
(0.33)\end{array}$ & $\begin{array}{l}0.219^{\mathrm{TW}} \\
(0.031)\end{array}$ & 0.012 & 232 & 81 \\
\hline $\begin{array}{l}\text { Number of under-five } \\
\text { deaths }\end{array}$ & $\begin{array}{l}0.011^{\mathrm{T \top}} \\
(0.002)\end{array}$ & $\begin{array}{l}0.011^{\mathrm{T \top}} \\
(0.009)\end{array}$ & 0.034 & 578 & 140 & $\begin{array}{c}0.183^{\mathrm{T \top}} \\
(0.53)\end{array}$ & $\begin{array}{c}0.242^{\mathrm{T}} \\
(0.47)\end{array}$ & 0.015 & 501 & 134 \\
\hline $\begin{array}{l}\text { Prevalence of } \\
\text { Undernourishment }(\% \\
\text { of population) }\end{array}$ & $\begin{array}{l}0.723^{\mp} \\
(0.711)\end{array}$ & $\begin{array}{l}0.274^{\mathrm{T \top}} \\
(13.77)\end{array}$ & 0.052 & 452 & 102 & $\begin{array}{c}3.295^{\mathrm{T}} \\
(0.51)\end{array}$ & $\begin{array}{l}0.297^{\mathrm{T \top}} \\
(0.449)\end{array}$ & 0.039 & 396 & 99 \\
\hline $\begin{array}{l}\text { Prevalence of } \\
\text { underweight }\end{array}$ & $\begin{array}{c}3.295^{\mathrm{T \top}} \\
(0.51)\end{array}$ & $\begin{array}{l}0.297^{\mathrm{T \top}} \\
(0.449)\end{array}$ & 0.035 & 391 & 89 & $\begin{array}{l}0.349^{\mathrm{T \top}} \\
(4.331)\end{array}$ & $\begin{array}{l}0.112^{\mathrm{T \top}} \\
(0.101)\end{array}$ & 0.019 & 331 & 90 \\
\hline Incidence of HIV & $\begin{array}{l}0.932^{\mathrm{T}} \\
(3.373)\end{array}$ & $\begin{array}{l}0.260^{\mathrm{T \top}} \\
(0.925)\end{array}$ & 0.086 & 501 & 144 & $\begin{array}{l}0.723^{\mathrm{T \top}} \\
(0.711)\end{array}$ & $\begin{array}{l}0.274^{\mathrm{T \top}} \\
(13.76)\end{array}$ & 0.096 & 445 & 130 \\
\hline Incidence of malaria & $\begin{array}{l}0.510^{\mathrm{T \top}} \\
(0.494)\end{array}$ & $\begin{array}{l}0.356^{\mathrm{T \top}} \\
(0.931)\end{array}$ & 0.01 & 352 & 111 & $\begin{array}{c}3.295^{\mathrm{T \top}} \\
(0.51)\end{array}$ & $\begin{array}{l}0.296^{\mathrm{T \top}} \\
(0.449)\end{array}$ & 0.058 & 391 & 109 \\
\hline $\begin{array}{l}\text { Incidence of } \\
\text { Tuberculosis }\end{array}$ & $\begin{array}{l}0.723^{\text {TФ }} \\
(0.711)\end{array}$ & $\begin{array}{l}0.274^{\mathrm{T \top}} \\
(13.77)\end{array}$ & 0.059 & 396 & 129 & $\begin{array}{l}4.254^{\mathrm{TT}} \\
(0.252)\end{array}$ & $\begin{array}{l}0.425^{\text {TஈT }} \\
(0.154)\end{array}$ & 0.023 & 334 & 115 \\
\hline
\end{tabular}

Note; Results shows the robust analysis using the ordinary least square. (e) and (r) columns show the values of independent variable i.e. ethnic and religious diversity respectively. Coff.. C, R-sqd., Obs, Cros.Sec shows the coefficient value, constant,

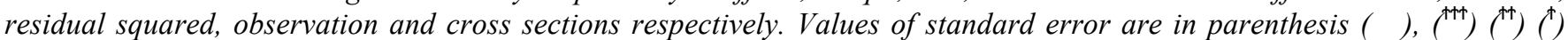
shows the level of significance at 1\%, 5\% and $10 \%$ respectively. 
Table 1c - Effects of ethnic and religious diversity on exclusion from civic and public participation

\begin{tabular}{|c|c|c|c|c|c|c|c|c|c|c|}
\hline \multirow{2}{*}{$\begin{array}{l}\text { Dependant } \\
\text { Variables }\end{array}$} & Coff. & $\mathrm{C}$ & $\begin{array}{c}\mathrm{R}- \\
\mathrm{sqd},\end{array}$ & Obs. & Cros.Sec & Coff. & $\mathrm{C}$ & R-sqd. & Obs. & Cros.Sec \\
\hline & (e) & (e) & (e) & (e) & (e) & (r) & (r) & $(\mathrm{r})$ & $(\mathrm{r})$ & $(\mathrm{r})$ \\
\hline $\begin{array}{l}\text { Vulnerable } \\
\text { employment }\end{array}$ & $\begin{array}{l}5.806^{\mathrm{T \top}} \\
(0.434)\end{array}$ & $\begin{array}{l}0.275^{\mathrm{T \top}} \\
(0.127)\end{array}$ & 0.093 & 376 & 90 & $\begin{array}{l}3.650^{\mathrm{T \top}} \\
(0.195)\end{array}$ & $\begin{array}{l}0.345^{\text {ॠ币 }} \\
(0.779)\end{array}$ & 0.036 & 294 & 88 \\
\hline Gender inequality & $\begin{array}{l}0.732^{\mathrm{T \top}} \\
(0.581)\end{array}$ & $\begin{array}{l}1.541^{\mathrm{T \top}} \\
(1.207)\end{array}$ & 0.025 & 564 & 127 & $\begin{array}{l}0.056^{\mathrm{T \top}} \\
(0.015)\end{array}$ & $\begin{array}{l}0.003^{\mathrm{T \top}} \\
(0.016)\end{array}$ & 0.093 & 419 & 117 \\
\hline $\begin{array}{l}\text { Lack of civil } \\
\text { Activism }\end{array}$ & $\begin{array}{l}0.516^{\text {ॠT }} \\
(0.601)\end{array}$ & $\begin{array}{l}0.220^{\mathrm{T}} \\
(0.313)\end{array}$ & 0.081 & 678 & 151 & $\begin{array}{l}0.462^{\text {Tஈ }} \\
(0.361)\end{array}$ & $\begin{array}{l}0.007^{\mathrm{T \top \textrm {T }}} \\
(0.051)\end{array}$ & 0.014 & 309 & 140 \\
\hline $\begin{array}{l}\text { Lack of intergroup } \\
\text { cohesion }\end{array}$ & $\begin{array}{l}1.259^{\mathrm{T \top}} \\
(0.119)\end{array}$ & $\begin{array}{l}0.080^{\top \mp \top} \\
(0.027)\end{array}$ & 0.030 & 658 & 149 & $\begin{array}{l}0.122^{\text {ॠT }} \\
(0.042)\end{array}$ & $\begin{array}{l}0.404^{\mathrm{T \top}} \\
(0.329)\end{array}$ & 0.022 & 588 & 151 \\
\hline $\begin{array}{l}\text { Less of club And } \\
\text { membership }\end{array}$ & $\begin{array}{l}1.855^{\mathrm{T \top}} \\
(0.354)\end{array}$ & $\begin{array}{l}0.103^{\text {TФ }} \\
(0.044)\end{array}$ & 0.015 & 580 & 141 & $\begin{array}{l}0.906^{\mp \top} \\
(0.967)\end{array}$ & $\begin{array}{l}0.698^{\mathrm{TФ}} \\
(0.751)\end{array}$ & 0.038 & 549 & 133 \\
\hline $\begin{array}{l}\text { Less safety and } \\
\text { trust }\end{array}$ & $\begin{array}{l}0.678^{\text {ॠФ }} \\
(0.338)\end{array}$ & $\begin{array}{l}0.774^{\text {ФФ }} \\
(0.625)\end{array}$ & 0.040 & 618 & 145 & $\begin{array}{c}0.210^{\mathrm{T \top}} \\
(0.69)\end{array}$ & $\begin{array}{l}0.254^{\mathrm{T \top}} \\
(0.454)\end{array}$ & 0.078 & 640 & 144 \\
\hline territory of asylum & $\begin{array}{l}0.519^{\text {ФФ }} \\
(0.745)\end{array}$ & $\begin{array}{l}0.463^{\text {ॠФ }} \\
(0.551)\end{array}$ & 0.062 & 388 & 97 & $\begin{array}{l}3.650^{\mp \mp \top} \\
(0.195)\end{array}$ & $\begin{array}{l}0.401^{\mathrm{T \top}} \\
(0.836)\end{array}$ & 0.042 & 330 & 92 \\
\hline Emigrants & $\begin{array}{l}0.254^{\mathrm{TT}} \\
(0.598)\end{array}$ & $\begin{array}{l}0.247^{\mathrm{TW}} \\
(0.429)\end{array}$ & 0.037 & 498 & 123 & $\begin{array}{l}1.978^{\mathrm{T \top}} \\
(0.048)\end{array}$ & $\begin{array}{l}0.003^{\mathrm{T \top}} \\
(0.008)\end{array}$ & 0.025 & 410 & 110 \\
\hline
\end{tabular}

Note; Results shows the robust analysis using the ordinary least square. (e) and ( $r$ ) columns show the values of independent variable i.e. ethnic and religious diversity respectively. Coff.. C, R-sqd., Obs, Cros.Sec shows the coefficient value, constant,

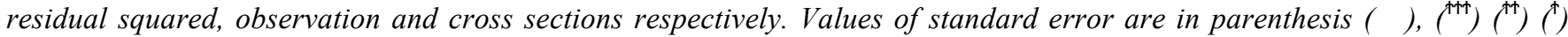
shows the level of significance at $1 \%, 5 \%$ and $10 \%$ respectively. 
Table 2a - Effects of ethnic and religious diversity on economic exclusion

\begin{tabular}{|c|c|c|c|c|c|c|c|c|}
\hline Variables & $\begin{array}{c}\text { Unemploy } \\
\text { ment } \\
\text { (UNEMP) }\end{array}$ & $\begin{array}{l}\text { Inequality } \\
\text { (GINI) }\end{array}$ & $\begin{array}{c}\text { Depth } \\
\text { Food } \\
\text { Deficit } \\
\text { (kilocalori } \\
\text { es) }\end{array}$ & $\begin{array}{c}\text { Poverty } \\
\text { (Head } \\
\text { count } \\
\text { Ratio) }\end{array}$ & $\begin{array}{c}\text { Poverty } \\
\text { (Gap } \\
\text { Ratio) }\end{array}$ & $\begin{array}{c}\text { Corruption } \\
\text { Score }\end{array}$ & $\begin{array}{c}\text { Intentional } \\
\text { Homicides } \\
\text { (per } \\
100,000 \\
\text { people) }\end{array}$ & $\begin{array}{l}\text { Out-of- } \\
\text { pocket } \\
\text { health } \\
\text { expendit } \\
\text { ure }\end{array}$ \\
\hline & (1) & (2) & (3) & (4) & (5) & (6) & $(7)$ & $(8)$ \\
\hline Ethnic Group & $\begin{array}{l}4.254^{\mathrm{\top}} \\
(5.806)\end{array}$ & $\begin{array}{l}0.024^{\mp \Phi} \\
(0.487)\end{array}$ & $\begin{array}{l}0.107^{\mathrm{T}} \\
(0.223)\end{array}$ & $\begin{array}{l}0.789^{\mp \top} \\
(1.324)\end{array}$ & $\begin{array}{l}0.160^{\mp \Phi} \\
(0.671)\end{array}$ & $\begin{array}{l}0.126^{\mp \Phi} \\
(0.019)\end{array}$ & $\begin{array}{l}0.100^{\mp \mp} \\
(0.018)\end{array}$ & $\begin{array}{c}0.467^{\Phi} \\
(0.500)\end{array}$ \\
\hline Religious Group & $\begin{array}{l}0.252^{\mathrm{TT}} \\
(0.434)\end{array}$ & $\begin{array}{l}0.352^{\mathrm{T}} \\
(0.104)\end{array}$ & $\begin{array}{l}-0.004 \\
(0.013)\end{array}$ & $\begin{array}{l}3.262^{\mathrm{TT}} \\
(1.405)\end{array}$ & $\begin{array}{c}0.152 \\
(0.019)\end{array}$ & $\begin{array}{l}-0.026^{\mp} \\
(0.022)\end{array}$ & $\begin{array}{l}0.068^{\text {TФ }} \\
(0.021)\end{array}$ & $\begin{array}{c}1.374^{\Phi} \\
(0.581)\end{array}$ \\
\hline GDPPC & $\begin{array}{c}0.425^{\mp} \\
(0.275)\end{array}$ & $\begin{array}{l}0.001^{\mathrm{T \top}} \\
(0.006)\end{array}$ & $\begin{array}{c}4.131^{\Phi} \\
(1.708)\end{array}$ & $\begin{array}{l}1.518^{\top \mathrm{T}} \\
(3.422)\end{array}$ & $\begin{array}{l}0.120^{\mp \top} \\
(0.286)\end{array}$ & $\begin{array}{l}0.401^{\text {ФТ }} \\
(0.203)\end{array}$ & $\begin{array}{c}-0.001^{\mathrm{T}} \\
(0.001)\end{array}$ & $\begin{array}{c}0.002 \\
(0.007)\end{array}$ \\
\hline Urbanization & $\begin{array}{c}0.154 \\
(0.127)\end{array}$ & $\begin{array}{l}-0.073 \\
(0.068)\end{array}$ & $\begin{array}{l}-0.003 \\
(0.006)\end{array}$ & $\begin{array}{c}0.011 \\
(0.041)\end{array}$ & $\begin{array}{c}0.412 \\
(0.922)\end{array}$ & $\begin{array}{l}-2.106 \\
(9.863)\end{array}$ & $\begin{array}{l}-6.131 \\
(9.26)\end{array}$ & $\begin{array}{c}0.406 \\
(0.202)\end{array}$ \\
\hline $\begin{array}{l}\text { Education } \\
\text { expenditure }\end{array}$ & $\begin{array}{l}-0.023 \\
(0.093)\end{array}$ & $\begin{array}{l}-0.008 \\
(0.052)\end{array}$ & $\begin{array}{l}-0.001 \\
(0.003)\end{array}$ & $\begin{array}{l}-0.016 \\
(0.011)\end{array}$ & $\begin{array}{l}-0.003 \\
(0.560)\end{array}$ & $\begin{array}{c}9.524 \\
(8.642)\end{array}$ & $\begin{array}{l}-4.000 \\
(1.532)\end{array}$ & $\begin{array}{c}0.003 \\
(0.002)\end{array}$ \\
\hline Pop. Density & $\begin{array}{c}0.046 \\
(0.176)\end{array}$ & $\begin{array}{l}-0.096 \\
(0.030)\end{array}$ & $\begin{array}{l}-0.004 \\
(0.003)\end{array}$ & $\begin{array}{l}-5.194 \\
(4.182)\end{array}$ & $\begin{array}{c}0.003 \\
(1.336)\end{array}$ & $\begin{array}{c}0.297 \\
(0.057)\end{array}$ & $\begin{array}{c}0.288^{\mp} \\
(0.053)\end{array}$ & $\begin{array}{c}0.429 \\
(1.451)\end{array}$ \\
\hline Health expenditure & $\begin{array}{l}-0.006 \\
(0.003)\end{array}$ & $\begin{array}{l}-1.373 \\
(0.255)\end{array}$ & $\begin{array}{l}-2.638 \\
(1.264)\end{array}$ & $\begin{array}{c}0.089 \\
(0.036)\end{array}$ & $\begin{array}{c}6.081 \\
(3.625)\end{array}$ & $\begin{array}{l}-0.006 \\
(0.002)\end{array}$ & $\begin{array}{l}-0.041 \\
(0.016)\end{array}$ & $\begin{array}{l}-0.026 \\
(0.012)\end{array}$ \\
\hline Literacy rate & $\begin{array}{l}-0.552 \\
(0.357)\end{array}$ & $\begin{array}{l}-0.002 \\
(0.012)\end{array}$ & $\begin{array}{c}1.016 \\
(2.773)\end{array}$ & $\begin{array}{l}-0.054 \\
(0.039)\end{array}$ & $\begin{array}{c}0.031 \\
(0.717)\end{array}$ & $\begin{array}{c}4.572 \\
(3.351)\end{array}$ & $\begin{array}{l}3.343^{\text {TT }} \\
(3.632)\end{array}$ & $\begin{array}{c}6.474 \\
(9.651)\end{array}$ \\
\hline $\begin{array}{l}\text { Institutional } \\
\text { Quality }\end{array}$ & $\begin{array}{l}-0.277 \\
(0.190)\end{array}$ & $\begin{array}{c}0.309 \\
(0.193)\end{array}$ & $\begin{array}{c}3.921 \\
(0.185)\end{array}$ & $\begin{array}{l}-0.545^{\mp} \\
(2.677)\end{array}$ & $\begin{array}{c}0.158 \\
(24.71)\end{array}$ & $\begin{array}{l}-0.560 \\
(0.310)\end{array}$ & $\begin{array}{l}-0.446 \\
(0.260)\end{array}$ & $\begin{array}{l}-0.005 \\
(0.012)\end{array}$ \\
\hline $\mathrm{C}$ & $\begin{array}{l}0.094^{\mathrm{\top} \mathrm{T}} \\
(0.221) \\
\end{array}$ & $\begin{array}{l}0.009^{\mathrm{T \top}} \\
(0.083) \\
\end{array}$ & $\begin{array}{c}0.007^{\mathrm{T \top}} \\
(0.116) \\
\end{array}$ & $\begin{array}{l}2.030^{\mp \top \mathrm{T}} \\
(2.635) \\
\end{array}$ & $\begin{array}{l}7.339^{\mp \top \mathrm{T}} \\
(2.908) \\
\end{array}$ & $\begin{array}{c}0.583^{\mp \mp \mathrm{T}} \\
(0.048) \\
\end{array}$ & $\begin{array}{l}0.513^{\mathrm{T \top}} \\
(0.046) \\
\end{array}$ & $\begin{array}{l}3.392^{\mathrm{T \top}} \\
(1.272) \\
\end{array}$ \\
\hline $\mathrm{N}$ & 315 & 256 & 216 & 246 & 270 & 272 & 253 & 267 \\
\hline$R^{2}$ & 0.247 & 0.344 & 0.269 & 0.241 & 0.181 & 0.251 & 0.284 & 0.091 \\
\hline Adj. $R^{2}$ & 0.198 & 0.309 & 0231 & 0.202 & 0.156 & 0.228 & 0.245 & 0.063 \\
\hline F-Stat & 1.64 & 9.67 & 7.22 & 6.24 & 7.24 & 11.03 & 7.16 & 3.24 \\
\hline
\end{tabular}

Notes: Number of proxy variable used to measure the economic exclusion as indicate by UNDP. Values of standard errors are in parentheses ()$.\left(^{\dagger+\dagger}\right)\left(^{\dagger+}\right)\left(^{\dagger}\right.$ shows the level of significance at $1 \%$, 5\% and $10 \%$ respectively. 
Table 2b - Effects of Ethnic Diversity on Exclusion from public services

\begin{tabular}{|c|c|c|c|c|c|c|c|}
\hline Variables & $\begin{array}{l}\text { Lack of } \\
\text { save } \\
\text { drinking } \\
\text { water }\end{array}$ & $\begin{array}{l}\text { Out of } \\
\text { school } \\
\text { children, }\end{array}$ & $\begin{array}{l}\text { Lifetime } \\
\text { risk of } \\
\text { maternal } \\
\text { death }(\%)\end{array}$ & $\begin{array}{c}\text { Low-birth } \\
\text { weight } \\
\text { babies (\% } \\
\text { of births) }\end{array}$ & $\begin{array}{c}\text { Maternal } \\
\text { mortality } \\
\text { ratio }\end{array}$ & $\begin{array}{l}\text { Mortality } \\
\text { rate, under- } \\
5\end{array}$ & $\begin{array}{c}\text { Number of } \\
\text { under-five } \\
\text { deaths }\end{array}$ \\
\hline & $(1)$ & $(2)$ & $(3)$ & $(4)$ & $(5)$ & $(6)$ & $(7)$ \\
\hline Ethnic Group & $\begin{array}{c}8.14^{\text {T}} \\
(1.344)\end{array}$ & $\begin{array}{l}0.053^{\mathrm{T}} \\
(0.025)\end{array}$ & $\begin{array}{l}8.347^{\mathrm{TT}} \\
(3.205)\end{array}$ & $\begin{array}{c}0.512^{\mathrm{T}} \\
(1.811)\end{array}$ & $\begin{array}{l}0.467^{\text {ॠT }} \\
(0.500)\end{array}$ & $\begin{array}{l}0.297^{\mathrm{T}} \\
(0.439)\end{array}$ & $\begin{array}{c}0.006^{\mathrm{T}} \\
(0.263)\end{array}$ \\
\hline Religious Group & $\begin{array}{c}1.166 \\
(1.576)\end{array}$ & $\begin{array}{l}0.043^{\text {TT }} \\
(0.030)\end{array}$ & $\begin{array}{l}8.475^{\text {tक }} \\
(3.480)\end{array}$ & $\begin{array}{c}1.993 \\
(2.095)\end{array}$ & $\begin{array}{c}1.374^{\oplus} \\
(0.581)\end{array}$ & $\begin{array}{c}1.682 \\
(0.520)\end{array}$ & $\begin{array}{c}0.816 \\
(0.308)\end{array}$ \\
\hline GDPPC & $\begin{array}{c}-0.073^{\text {बФ帀 }} \\
(0.021)\end{array}$ & $\begin{array}{c}-0.001^{\mathrm{T}} \\
(0.003)\end{array}$ & $\begin{array}{l}-0.119^{\mathrm{T}} \\
(0.045)\end{array}$ & $\begin{array}{c}-0.028^{\mathrm{T}} \\
(0.027)\end{array}$ & $\begin{array}{l}-0.002^{\mathrm{T}} \\
(0.007)\end{array}$ & $\begin{array}{c}-0.210^{\mathrm{TW}} \\
(0.160)\end{array}$ & $\begin{array}{c}-0.001^{\text {बФТ }} \\
(0.004)\end{array}$ \\
\hline Urbanization & $\begin{array}{l}-0.012 \\
(0.006)\end{array}$ & $\begin{array}{c}0.406 \\
(0.210)\end{array}$ & $\begin{array}{c}0.012 \\
(0.011)\end{array}$ & $\begin{array}{l}-0.016 \\
(0.008)\end{array}$ & $\begin{array}{c}0.010 \\
(0.012)\end{array}$ & $\begin{array}{l}-0.001 \\
(0.002)\end{array}$ & $\begin{array}{l}-0.003 \\
(0.001)\end{array}$ \\
\hline Education expenditure & $\begin{array}{c}-0.002 \\
(0.006)\end{array}$ & $\begin{array}{c}-4.121^{\mathrm{T}} \\
(1.340)\end{array}$ & $\begin{array}{c}-0.034 \\
(0.016)\end{array}$ & $\begin{array}{c}0.024 \\
(0.008)\end{array}$ & $\begin{array}{c}0.003 \\
(0.002)\end{array}$ & $\begin{array}{c}0.010 \\
(0.002)\end{array}$ & $\begin{array}{c}0.015 \\
(0.001)\end{array}$ \\
\hline Pop. Density & $\begin{array}{c}13.77 \\
(3.975)\end{array}$ & $\begin{array}{l}-0.039 \\
(0.081)\end{array}$ & $\begin{array}{l}-13.95 \\
(9.724)\end{array}$ & $\begin{array}{l}-4.199 \\
(5.404)\end{array}$ & $\begin{array}{l}0.429^{\mathrm{TT}} \\
(1.451)\end{array}$ & $\begin{array}{c}2.088 \\
(1.301)\end{array}$ & $\begin{array}{c}0.822 \\
(0.778)\end{array}$ \\
\hline Health expenditure & $\begin{array}{l}-0.033 \\
(0.035)\end{array}$ & $\begin{array}{l}-0.360 \\
(0.160)\end{array}$ & $\begin{array}{l}-0.500 \\
(0.275)\end{array}$ & $\begin{array}{c}-0.013 \\
(0.045)\end{array}$ & $\begin{array}{l}-0.026 \\
(0.012)\end{array}$ & $\begin{array}{c}-0.033 \\
(0.011)\end{array}$ & $\begin{array}{c}-0.008 \\
(0.006)\end{array}$ \\
\hline Literacy rate & $\begin{array}{c}-4.370 \\
(1.704)\end{array}$ & $\begin{array}{c}-1.097^{\mathrm{T \top}} \\
(0.112)\end{array}$ & $\begin{array}{c}-0.200 \\
(0.110)\end{array}$ & $\begin{array}{c}-3.021^{\mathrm{T}} \\
(4.226)\end{array}$ & $\begin{array}{c}6.473 \\
(9.652)\end{array}$ & $\begin{array}{c}-8.404^{\mathrm{T \top}} \\
(8.744)\end{array}$ & $\begin{array}{c}7.087 \\
(5.283)\end{array}$ \\
\hline Institutional Quality & $\begin{array}{c}-0.084 \\
(0.031)\end{array}$ & $\begin{array}{c}-0.001^{\mathrm{TT}} \\
(0.002)\end{array}$ & $\begin{array}{c}0.409 \\
(0.070)\end{array}$ & $\begin{array}{l}-0.120 \\
(0.041)\end{array}$ & $\begin{array}{l}-0.005 \\
(0.012)\end{array}$ & $\begin{array}{l}-0.022 \\
(0.010)\end{array}$ & $\begin{array}{l}-0.005 \\
(0.006)\end{array}$ \\
\hline $\mathrm{C}$ & $\begin{array}{l}69.32^{\text {ॠФ }} \\
(3.470)\end{array}$ & $\begin{array}{c}0.428^{\text {ФФ }} \\
(0.064)\end{array}$ & $\begin{array}{c}43.60^{\mathrm{T \top}} \\
(7.285)\end{array}$ & $\begin{array}{c}21.05^{\top \top \top} \\
(4.553)\end{array}$ & $\begin{array}{l}3.392^{\mathrm{T \top}} \\
(1.272)\end{array}$ & $\begin{array}{c}4.953^{\top \top} \\
(1.131)\end{array}$ & $\begin{array}{l}3.182^{\text {ॠТ }} \\
(0.679)\end{array}$ \\
\hline $\mathrm{N}$ & 273 & 310 & 245 & 217 & 272 & 256 & 275 \\
\hline$R^{2}$ & 0.122 & 0.385 & 0.191 & 0.163 & 0.241 & 0.162 & 0.142 \\
\hline Adj. $R^{2}$ & 0.095 & 0.351 & 0.164 & 0.123 & 0.218 & 0.131 & 0.120 \\
\hline F-Stat & 4.59 & 7.01 & 7.00 & 4.09 & 10.46 & 2.04 & 16.3 \\
\hline
\end{tabular}

Notes: Number of proxy variable used to measure the economic exclusion as indicate by UNDP. Values of standard errors are in parentheses ()$\left..{ }^{\dagger+t}\right)\left(^{\dagger+}\right)\left(^{\dagger}\right.$ shows the level of significance at $1 \%$, 5\% and $10 \%$ respectively. 
Table 2c- Effects of Ethnic Diversity on Exclusion from public services

\begin{tabular}{|c|c|c|c|c|c|c|c|}
\hline Variables & $\begin{array}{l}\text { Prevalence } \\
\text { of } \\
\text { Undernouri } \\
\text { shment } \\
\text { (\% of } \\
\text { population) }\end{array}$ & $\begin{array}{c}\text { Prevalence } \\
\text { of } \\
\text { Underweigh } \\
t\end{array}$ & $\begin{array}{l}\text { Children } \\
\text { living } \\
\text { with HIV }\end{array}$ & $\begin{array}{l}\text { Contracep } \\
\text { tive } \\
\text { Prevalenc } \\
\text { e }\end{array}$ & $\begin{array}{c}\text { Incidence } \\
\text { of } \\
\text { HIV }\end{array}$ & $\begin{array}{c}\text { Incidence } \\
\text { of } \\
\text { Malaria }\end{array}$ & $\begin{array}{l}\text { Incidence } \\
\text { of } \\
\text { Tuberculos } \\
\text { is }\end{array}$ \\
\hline & $(8)$ & (8) & $(10)$ & (11) & $(12)$ & (13) & (14) \\
\hline Ethnic Group & $\begin{array}{l}0.304^{\mathrm{T}} \\
(1.273)\end{array}$ & $\begin{array}{c}0.999 \\
(0.986)\end{array}$ & $\begin{array}{c}0.992^{\mathrm{T}} \\
(0.818)\end{array}$ & $\begin{array}{l}0.796^{\text {Фक }} \\
(5.102)\end{array}$ & $\begin{array}{l}0.834^{\text {क巾 }} \\
(4.004)\end{array}$ & $\begin{array}{c}1.895 \\
(1.563)\end{array}$ & $\begin{array}{r}0.055 \\
(0.025)\end{array}$ \\
\hline Religious Group & $\begin{array}{c}0.300 \\
(1.260)\end{array}$ & $\begin{array}{c}1.535^{\mathrm{T}} \\
(2.098)\end{array}$ & $\begin{array}{c}1.557 \\
(2.009)\end{array}$ & $\begin{array}{l}0.506^{\mathrm{T \top}} \\
(3.444)\end{array}$ & $\begin{array}{c}0.579 \\
(3.191)\end{array}$ & $\begin{array}{c}0.754 \\
(0.286)\end{array}$ & $\begin{array}{c}0.031^{\mathrm{\Phi}} \\
(0.030)\end{array}$ \\
\hline GDPPC & $\begin{array}{l}-0.708^{\mathrm{T}} \\
(0.324)\end{array}$ & $\begin{array}{c}-1.743^{\top \Phi} \\
(3.063)\end{array}$ & $\begin{array}{c}-0.403 \\
(0.749)\end{array}$ & $\begin{array}{l}-0.457^{\mathrm{T}} \\
(1.451)\end{array}$ & $\begin{array}{c}-0.209 \\
(0.564)\end{array}$ & $\begin{array}{l}-0.399 \\
(0.463)\end{array}$ & $\begin{array}{r}-0.001 \\
(0.001)\end{array}$ \\
\hline Urbanization & $\begin{array}{l}-1.217 \\
(1.55)\end{array}$ & $\begin{array}{l}-2.581 \\
(4.680)\end{array}$ & $\begin{array}{l}-0.180 \\
(0.029)\end{array}$ & $\begin{array}{l}-1.406 \\
(3.045)\end{array}$ & $\begin{array}{l}-0.850 \\
(2.073)\end{array}$ & $\begin{array}{c}0.010^{\mp} \\
(0.057)\end{array}$ & $\begin{array}{r}2.601 \\
(0.683)\end{array}$ \\
\hline Education expenditure & $\begin{array}{r}-1.175 \\
(2.77)\end{array}$ & $\begin{array}{l}-1.007 \\
(2.420)\end{array}$ & $\begin{array}{c}-0.832 \\
(0.642)\end{array}$ & $\begin{array}{l}-0.129 \\
(0.325)\end{array}$ & $\begin{array}{c}-0.193 \\
(0.448)\end{array}$ & $\begin{array}{c}0.136 \\
(0.251)\end{array}$ & $\begin{array}{r}-0.108 \\
(0.540)\end{array}$ \\
\hline Pop. Density & $\begin{array}{l}-0.998 \\
(3.019)\end{array}$ & $\begin{array}{l}-2.444 \\
(3.910)\end{array}$ & $\begin{array}{c}0.869 \\
(0.927)\end{array}$ & $\begin{array}{l}-2.661 \\
(5.801)\end{array}$ & $\begin{array}{c}-1.84 \\
(4.249)\end{array}$ & $\begin{array}{l}-0.416 \\
(0.682)\end{array}$ & $\begin{array}{r}-0.022 \\
(0.075)\end{array}$ \\
\hline Health expenditure & $\begin{array}{c}1.111 \\
(1.974)\end{array}$ & $\begin{array}{c}1.858 \\
(4.148)\end{array}$ & $\begin{array}{c}0.301 \\
(0.645)\end{array}$ & $\begin{array}{c}1.872 \\
(4.778)\end{array}$ & $\begin{array}{c}1.345 \\
(3.704)\end{array}$ & $\begin{array}{c}0.677 \\
(1.271)\end{array}$ & $\begin{array}{r}-0.220 \\
(0.114)\end{array}$ \\
\hline Literacy rate & $\begin{array}{c}1.620^{\mathrm{T}} \\
(2.964)\end{array}$ & $\begin{array}{c}1.878 \\
(2.133)\end{array}$ & $\begin{array}{c}1.565 \\
(3.012)\end{array}$ & $\begin{array}{c}-1.421^{\text {ФТ }} \\
(2.453)\end{array}$ & $\begin{array}{l}1.52^{\mathrm{T \top}} \\
(3.003)\end{array}$ & $\begin{array}{l}1.318^{\mathrm{T}} \\
(2.294)\end{array}$ & $\begin{array}{r}6.905 \\
(5.145)\end{array}$ \\
\hline Institutional Quality & $\begin{array}{l}-1.479 \\
(6.554)\end{array}$ & $\begin{array}{l}-1.383 \\
(5.856)\end{array}$ & $\begin{array}{c}-1.64 \\
(5.302)\end{array}$ & $\begin{array}{l}-2.190 \\
(5.073)\end{array}$ & $\begin{array}{c}-1.95 \\
(5.947)\end{array}$ & $\begin{array}{l}-1.725 \\
(4.207)\end{array}$ & $\begin{array}{r}0.451 \\
(0.224)\end{array}$ \\
\hline $\mathrm{C}$ & $\begin{array}{c}0.533^{\mathrm{T \top}} \\
(1.202)\end{array}$ & $\begin{array}{l}1.068^{\mathrm{T \top}} \\
(2.221)\end{array}$ & $\begin{array}{c}-1.670^{\mathrm{ФФ}} \\
(2.769) \\
\end{array}$ & $\begin{array}{c}-0.308^{\mathrm{TФ}} \\
(0.512) \\
\end{array}$ & $\begin{array}{c}-0.081^{\text {ФФ }} \\
(0.139) \\
\end{array}$ & $\begin{array}{l}0.816^{\mathrm{\top Ф}} \\
(1.084)\end{array}$ & $\begin{array}{c}0.006^{\mathrm{TT}} \\
(0.003)\end{array}$ \\
\hline $\begin{array}{l}\mathrm{N} \\
R^{2} \\
\text { Adj. } R^{2} \\
\text { F-Stat }\end{array}$ & $\begin{array}{c}256 \\
0.087 \\
0.058 \\
2.97\end{array}$ & $\begin{array}{c}233 \\
0.166 \\
0.135 \\
5.33\end{array}$ & $\begin{array}{c}251 \\
0.344 \\
0.309 \\
9.67\end{array}$ & $\begin{array}{c}239 \\
0.351 \\
0.325 \\
17.38\end{array}$ & $\begin{array}{c}298 \\
0.516 \\
0.487 \\
9.41\end{array}$ & $\begin{array}{c}273 \\
0.122 \\
0.095 \\
4.59\end{array}$ & $\begin{array}{c}262 \\
0.144 \\
0.117 \\
5.33\end{array}$ \\
\hline
\end{tabular}

Notes: Number of proxy variable used to measure the exclusion from public services as indicate by UNDP. Values

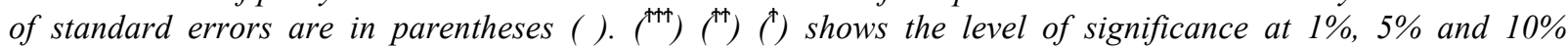
respectively. 
Table 2d - Effects of Ethnic Diversity on Exclusion from civic and public participation

\begin{tabular}{|c|c|c|c|c|c|c|c|c|}
\hline Variables & $\begin{array}{l}\text { Vulnerabl } \\
\text { e } \\
\text { Employm } \\
\text { ent }\end{array}$ & $\begin{array}{l}\text { Gender } \\
\text { inequality }\end{array}$ & $\begin{array}{l}\text { Lack of } \\
\text { civil } \\
\text { Activism }\end{array}$ & $\begin{array}{c}\text { Lack of } \\
\text { intergroup } \\
\text { cohesion }\end{array}$ & $\begin{array}{c}\text { Less of } \\
\text { club } \\
\text { And } \\
\text { members } \\
\text { hip }\end{array}$ & $\begin{array}{c}\text { Less safety } \\
\text { and } \\
\text { trust }\end{array}$ & $\begin{array}{l}\text { territory of } \\
\text { asylum }\end{array}$ & Emigrants \\
\hline & (1) & (2) & (3) & (4) & (5) & (6) & $(7)$ & $(8)$ \\
\hline Ethnic Group & $\begin{array}{c}0.050^{\mp} \\
(0.123)\end{array}$ & $\begin{array}{l}4.474^{\mathrm{T}} \\
(2.097)\end{array}$ & $\begin{array}{l}0.140^{\mp \Phi} \\
(0.5174)\end{array}$ & $\begin{array}{c}0.159^{\mp \Phi} \\
(0.5982)\end{array}$ & $\begin{array}{l}0.233^{\Phi} \\
(0.807)\end{array}$ & $\begin{array}{l}7.048^{\mathrm{T \top}} \\
(2.290)\end{array}$ & $\begin{array}{c}2.174^{\mathrm{T}} \\
(0.025)\end{array}$ & $\begin{array}{c}0.676^{\text {}} \\
(0.933)\end{array}$ \\
\hline Religious Group & $\begin{array}{l}0.196^{\text {ॠT }} \\
(0.385)\end{array}$ & $\begin{array}{c}4.454 \\
(3.796)\end{array}$ & $\begin{array}{l}0.112^{\mathrm{T \Phi}} \\
(0.340)\end{array}$ & $\begin{array}{l}0.463^{\text {ТФ }} \\
(1.453)\end{array}$ & $\begin{array}{c}0.360^{\mp} \\
(0.974)\end{array}$ & $\begin{array}{c}0.647^{\mathrm{T}} \\
(0.396)\end{array}$ & $\begin{array}{l}1.041^{\mathrm{T}} \\
(2.644)\end{array}$ & $\begin{array}{r}3.231 \\
(2.362)\end{array}$ \\
\hline GDPPC & $\begin{array}{l}-9.180^{\top} \\
(4.011)\end{array}$ & $\begin{array}{c}9.807 \\
(5.080)\end{array}$ & $\begin{array}{c}9.210 \\
(2.810)\end{array}$ & $\begin{array}{l}-6.530^{\mathrm{T}} \\
(3.326)\end{array}$ & $\begin{array}{l}-4.869^{\mathrm{T}} \\
(2.447)\end{array}$ & $\begin{array}{c}5.416 \\
(1.037)\end{array}$ & $\begin{array}{r}-4.354 \\
(0.438)\end{array}$ & $\begin{array}{r}-0.094 \\
(0.357)\end{array}$ \\
\hline Urbanization & $\begin{array}{c}0.194 \\
(0.179)\end{array}$ & $\begin{array}{l}-0.033 \\
(0.046)\end{array}$ & $\begin{array}{l}-0.266 \\
(0.298)\end{array}$ & $\begin{array}{l}-0.155 \\
(0.260)\end{array}$ & $\begin{array}{l}-0.515 \\
(0.802)\end{array}$ & $\begin{array}{c}1.028^{\mathrm{T}} \\
(1.294)\end{array}$ & $\begin{array}{r}-0.197 \\
(-2.715)\end{array}$ & $\begin{array}{r}-0.517 \\
(0.355)\end{array}$ \\
\hline $\begin{array}{l}\text { Education } \\
\text { expenditure }\end{array}$ & $\begin{array}{l}-1.328 \\
(2.047)\end{array}$ & $\begin{array}{l}-1.743 \\
(2.989)\end{array}$ & $\begin{array}{l}-0.328 \\
(0.235)\end{array}$ & $\begin{array}{l}-1.221 \\
(2.039)\end{array}$ & $\begin{array}{l}-1.063 \\
(1.658)\end{array}$ & $\begin{array}{l}-1.050 \\
(0.829)\end{array}$ & $\begin{array}{r}-2.145 \\
(12.37)\end{array}$ & $\begin{array}{r}5.042 \\
(1.626)\end{array}$ \\
\hline Pop. Density & $\begin{array}{c}0.398 \\
(0.402)\end{array}$ & $\begin{array}{l}-0.516 \\
(0.452)\end{array}$ & $\begin{array}{c}2.382 \\
(2.114)\end{array}$ & $\begin{array}{c}0.131 \\
(0.277)\end{array}$ & $\begin{array}{c}0.493 \\
(0.755)\end{array}$ & $\begin{array}{l}0.2235 \\
(0.209)\end{array}$ & $\begin{array}{r}-0.291 \\
(1.055)\end{array}$ & $\begin{array}{r}1.604 \\
(0.295)\end{array}$ \\
\hline Health expenditure & $\begin{array}{l}-1.397 \\
(1.774)\end{array}$ & $\begin{array}{c}-1.568 \\
(2.91)\end{array}$ & $\begin{array}{l}-0.291 \\
(0.252)\end{array}$ & $\begin{array}{l}-1.874 \\
(1.631)\end{array}$ & $\begin{array}{l}-0.714 \\
(0.994)\end{array}$ & $\begin{array}{l}0.1924 \\
(0.191)\end{array}$ & $\begin{array}{r}-0.837 \\
(1.271)\end{array}$ & $\begin{array}{r}-2.948 \\
(2.074)\end{array}$ \\
\hline Literacy rate & $\begin{array}{l}2.558^{\mathrm{T}} \\
(3.234)\end{array}$ & $\begin{array}{c}1.27 \\
(2.461)\end{array}$ & $\begin{array}{c}3.091 \\
(2.881)\end{array}$ & $\begin{array}{c}1.052 \\
(1.251)\end{array}$ & $\begin{array}{c}1.994 \\
(2.282)\end{array}$ & $\begin{array}{c}3.101 \\
(2.468)\end{array}$ & $\begin{array}{r}0.013 \\
(1.341)\end{array}$ & $\begin{array}{r}-0.960 \\
(0.670)\end{array}$ \\
\hline Institutional Quality & $\begin{array}{l}-2.962^{\mathrm{T}} \\
(9.635)\end{array}$ & $\begin{array}{l}-3.181^{\mathrm{T}} \\
(9.437)\end{array}$ & $\begin{array}{l}-2.516^{\mathrm{TT}} \\
(7.511)\end{array}$ & $\begin{array}{l}-2.549^{\mp} \\
(5.463)\end{array}$ & $\begin{array}{l}-2.74 \\
(5.95)\end{array}$ & $\begin{array}{l}-3.499^{\mathrm{T}} \\
(5.501)\end{array}$ & $\begin{array}{r}-1.949 \\
(0.962)\end{array}$ & $\begin{array}{c}-2.207^{\mp \Phi} \\
(0.453)\end{array}$ \\
\hline $\mathrm{C}$ & $\begin{array}{l}2.582^{\text {ФТ }} \\
(5.145) \\
\end{array}$ & $\begin{array}{l}2.922^{\mathrm{T \top}} \\
(5.032) \\
\end{array}$ & $\begin{array}{l}1.839^{\mathrm{T \top}} \\
(3.375) \\
\end{array}$ & $\begin{array}{l}2.022^{\mathrm{T \top}} \\
(2.488) \\
\end{array}$ & $\begin{array}{l}2.633^{\mathrm{T \top}} \\
(3.141) \\
\end{array}$ & $\begin{array}{l}4.531^{\mathrm{\top}} \\
(3.384) \\
\end{array}$ & $\begin{array}{c}14.47^{\text {ФФ }} \\
(0.958) \\
\end{array}$ & $\begin{array}{r}3.462^{\text {ТФ }} \\
(2.573) \\
\end{array}$ \\
\hline $\mathrm{N}$ & 251 & 262 & 264 & 291 & 270 & 267 & 273 & 224 \\
\hline$R^{2}$ & 0.263 & 0.485 & 0.339 & 0.420 & 0.526 & 0.395 & 0.234 & 0.402 \\
\hline Adj. $R^{2}$ & 0.223 & 0.418 & 0.297 & 0.346 & 0.473 & 0.324 & 0.152 & 0.337 \\
\hline F-Stat & 6.57 & 7.27 & 4.12 & 5.46 & 9.86 & 5.52 & 2.84 & 6.24 \\
\hline
\end{tabular}

Notes: Number of proxy variable used to measure the exclusion from civic and public participation as indicate by UNDP. Values of standard errors are in parentheses ( ). ${\left({ }^{\dagger+}\right)}^{\left.\dagger^{\dagger}\right)}\left(^{\dagger}\right)$ shows the level of significance at $1 \%$, 5\% and $10 \%$ respectively. 
Table 3 - Effects of Diversity on Social Exclusion ${ }^{4}$

\begin{tabular}{|c|c|c|c|}
\hline Variables & $\begin{array}{l}\text { Index for Economic } \\
\text { Exclusion }\end{array}$ & $\begin{array}{l}\text { Index for exclusion } \\
\text { of public service }\end{array}$ & $\begin{array}{c}\text { Index for exclusion of } \\
\text { civic and public } \\
\text { participation }\end{array}$ \\
\hline & $(1)$ & (2) & (3) \\
\hline Ethnic Group & $\begin{array}{l}0.314^{\mathrm{TfT}} \\
(0.148)\end{array}$ & $\begin{array}{l}0.100^{\mathrm{th}} \\
(0.150)\end{array}$ & $\begin{array}{l}0.154^{+7 \mathrm{~T}} \\
(0.956)\end{array}$ \\
\hline Religious Group & $\begin{array}{l}0.116^{Ð \uparrow} \\
(0.019)\end{array}$ & $\begin{array}{l}0.127^{\mathrm{T \top 巾}} \\
(0.050)\end{array}$ & $\begin{array}{l}0.013^{Ð \mathrm{~T}} \\
(0.153)\end{array}$ \\
\hline GDPPC & $\begin{array}{c}-0.002^{\mathrm{WT}} \\
(0.020)\end{array}$ & $\begin{array}{l}-0.047^{\mp} \\
(0.049)\end{array}$ & $\begin{array}{l}-0.153^{\mathrm{fT}} \\
(0.018)\end{array}$ \\
\hline Urbanization & $\begin{array}{c}0.951 \\
(0.603)\end{array}$ & $\begin{array}{c}0.153 \\
(0.377)\end{array}$ & $\begin{array}{c}5.512 \\
(7.310)\end{array}$ \\
\hline Edu. expenditure & $\begin{array}{l}-0.190^{\ddagger} \\
(0.100)\end{array}$ & $\begin{array}{l}-0.150 \\
(0.368)\end{array}$ & $\begin{array}{l}-0.048^{\mathrm{TP}} \\
(0.079)\end{array}$ \\
\hline Pop. Density & $\begin{array}{l}8.625 \\
(2.694)\end{array}$ & $\begin{array}{l}-1.252 \\
(0.242)\end{array}$ & $\begin{array}{l}10.082 \\
(4.687)\end{array}$ \\
\hline Health expenditure & $\begin{array}{l}-3.791 \\
(4.382)\end{array}$ & $\begin{array}{l}-0.055 \\
(0.166)\end{array}$ & $\begin{array}{l}-0.265 \\
(0.186)\end{array}$ \\
\hline Literacy rate & $\begin{array}{l}-2.238 \\
(7.258)\end{array}$ & $\begin{array}{l}-1.653^{\mathrm{T}} \\
(0.272)\end{array}$ & $\begin{array}{l}-2.762 \\
(5.480)\end{array}$ \\
\hline Institutional Quality & $\begin{array}{l}-6.314^{\mathrm{TP}} \\
(4.078)\end{array}$ & $\begin{array}{c}-0.011^{\mathrm{ffT}} \\
(0.154)\end{array}$ & $\begin{array}{l}-0.154^{\mathrm{fT}} \\
(2.952)\end{array}$ \\
\hline $\mathrm{C}$ & $\begin{array}{l}0.111^{\mathrm{fWT}} \\
(0.019)\end{array}$ & $\begin{array}{l}0.027^{\mathrm{ffT}} \\
(0.070)\end{array}$ & $\begin{array}{l}0.019^{\mathrm{ftP}} \\
(0.0143)\end{array}$ \\
\hline $\mathrm{N}$ & 267 & 296 & 266 \\
\hline$R^{2}$ & 0.395 & 0.526 & 0.402 \\
\hline Adj. $R^{2}$ & 0.324 & 0.473 & 0.337 \\
\hline F-Stat & 5.52 & 9.86 & 6.24 \\
\hline
\end{tabular}

Notes: Indices of social exclusion is created by using PCA technique. However, social exclusion is comprised on index of economic exclusion, index for exclusion of public service and the exclusion of civic and public participation as indicated by UNDP. Values of standard errors are in parentheses ()$.\left(^{(t+}\right)\left(^{+\top}\right)\left(^{+}\right)$shows the level of significance at $1 \%, 5 \%$ and $10 \%$ respectively.

The results indicate that diversity either ethnic or religious present enormous obstacles in development processes and variously contribute to social exclusion. In above tables 1a-1c and $2 a-2 d$ (using different proxies of each indicator of social exclusion) a robust relationship

\footnotetext{
${ }^{4}$ In order to check the relationship between diversity and social exclusion, results show robust analysis by using ordinary least square (OLS), fixed effect and random effect. Here the table shows only OLS results for ease of understanding for the reader.
} 
between diversity and social exclusion emerges using data from 187-countries. However, table-3 shows the direct effect of diversity on social exclusion (by created indices of economic exclusion, exclusion from public services and exclusion from civic and pubic participations). The results indicate that diversity (ethnic and religious) has a significant positive impact on social exclusion. Becker (1962) illustrates theoretically that discrimination in society can create prejudice that could lead to lower outcomes. The results are more illuminating in the regards that diversity appears to cause not only economic exclusion but also results in the exclusion of individuals from public services and civic/public participations. However, there are obviously numerous explanations for how diversity contributes toward social exclusion worldwide.

Firstly, higher diversity increases chances of conflict within society and the market place which ultimately results in low income, less economic development and low institutional quality (Alesina \& Rodrik, 1994; Alesina \& Spolaore, 1997; Alesina \& Tabellini, 1989; Easterly \& Levine, 1997; La Porta, Lopez-de-Silanes, Shleifer, \& Vishny, 1999; A. Sutherland, 1997). Secondly, ethnically polarized societies have difficulty agreeing on public goods like infrastructure, education, and public policies. It further brings about two fundamental setbacks, which are endemic diseases for development, i.e. rent- seeking activities and incongruity on public policies (Easterly \& Levine, 1997). Thirdly, ethnic and regional competition tends to degrade the institutional foundations of the economy such as when ethnic and personal attachments are the leading principle rather than the rule of law, ultimately contributing toward the deterioration of public institutional capacity (Nafziger \& Auvinen, 2003). These results are similar to Delhey and Newton (2005) and Dincer and Wang (2011) where they argued that diversity diminishes economic development and quality of institution because the ethnocentric members of an ethnic group favour their group members over others (Glaeser \& Saks, 2006; Nafziger \& Auvinen, 2003; Treisman, 2000; Van den Berghe, 1994).

Appasamy, Guhan, Hema, Majumdar, and Vaidyanathan (1996) indicate religious diversity is one of the prominent indicators of social exclusion. Bardsley and Flatley (1998) have argued that diversity in form of social class race, religious and ethnicity results in more exclusion for individuals denied equality of opportunity in areas of education, health, employment, basic needs and enjoyment of life. The current study's findings support this conclusion. People belonging to certain groups are disproportionately excluded from the benefits of achieving their full human potential and enjoying dignity and social standing.

Mason, MacGillivray, Steel, and Wilson (2003) have produced a step by step guide to community sustainability indicators and found that diversity has direct impact on socioeconomic development because it impacts civil society, social development and institutional performance of a country. Socially excluded people are more likely to be involved in breaking institutional rules (formal and informal) because they are not treated equal in society in all aspects and bypass the rules, regulations and social norms (Ananiev, Atanasov, GerovskaMitev, \& Shukarov, 2011; Mathieson et al., 2008).

Other control variables such as GDP per capita, education expenditure and institutional quality show significant impact on social exclusion. These results are supported by the literature as increasing the GDP per capital, educational expenditure and institutional quality reduced the effect of social exclusion (Lechman, 2014; Levitas et al., 2007; J. Sutherland, 2001). 


\section{Conclusion and policy implication}

This study aimed to show the relationship between diversity (ethnic and religious) and different dimensions of social exclusion, i.e. economic exclusion, exclusion from public services and civic/public participation, using data from 187-countries of the world. In order to judge the nexus between dependent and independent variables, panel data methodology was used in empirical analysis. The results elucidate the strong significant positive relationship between diversity and social exclusion and the need to redress this if the aim is to avoid creating more conflict among diversified groups which in turn, undermine society and institutional quality (Easterly, Ritzen, \& Woolcock, 2006). Diversity accompanied by weak institutions divides the society posing risks for religious and cultural confliction, civil wars, social tensions, political violence and unrest, corruption and are a recipe for underdevelopment (Alesina \& Rodrik, 1994; Alesina \& Spolaore, 1997; Alesina \& Tabellini, 1989; Easterly \& Levine, 1997; La Porta et al., 1999; A. Sutherland, 1997). The literature also shows diversity can create weak public institutions since 'elites' or those in positions of privilege have no time or willingness to contribute towards the growth of the national economy to reduce inequalities (Keen, 2000; Väyrynen, 2003). In this contemporary world, multi-ethnic cultural states of different races, colour, language, religion and regions are commonplace. Hence, diversity has important implications to improve social development globally. The findings of this study demonstrate that diversity is another important and significant variable affecting socioeconomic development and suggests that economies can prosper by better managing heterogeneity.

This study acknowledges that diversity cannot be reduced; however, its effects can be minimized by providing equal opportunities to all the individuals of the society, in order to create a secure and peaceful society through shaping the economic life of a country in a variety of ways and by promoting more cohesiveness. In this regard, there are some lessons to be learned from countries where there are numerous religions, race and cultures celebrated. For instance, celebrating various religious/festive days and showing respect for each other in public forums. Although, achieving diversity and respect for people is a challenge all over the world, there is also good progress being made in some corners of the world.

\section{Limitations and Prospects for Future Researches}

Every study has some limitations which require researchers to interpret the results within certain parameters. This study has also its limitations regarding limiting scope to consider the relationship between diversity and social exclusion. In addition to this, diversity is a multidimensional concept, i.e., demographic, socioeconomic, political, geographical cultural and dynamic in nature, so this study does not claim that the variables included are the only determinants or predictors of social exclusion and diversity. This study has also some limitations regarding the weakness of data on social exclusion, because this study used different proxy measures to undertake an empirical analysis of social exclusion.

The study of social exclusion is a vast field. Future research may seek to analyze some other factors (e.g. judicial, deterrence, ecological) that are related to diversity and social exclusion. Future researchers may also seek to collect their own data instead of using secondary data which may increase the reliability and validity of findings and reveal other socioeconomic determinants of exclusion. This study uses diversity on the basis of ethnic and religious differences only, whereas other determinants of diversity such as gender, may be opportunities for further research. It is also acknowledged that the current study did not include any political or ideological variables. 


\section{References}

Aasland, A., \& Fløtten, T. (2001). Ethnicity and social exclusion in Estonia and Latvia. Europe-Asia Studies, 53(7), 1023-1049.

Alesina, A., Devleeschauwer, A., Easterly, W., Kurlat, S., \& Wacziarg, R. (2003). Fractionalization. Journal of Economic growth, 8(2), 155-194.

Alesina, A., \& Rodrik, D. (1994). Distributive politics and economic growth. The Quarterly Journal of Economics, 109(2), 465-490.

Alesina, A., \& Spolaore, E. (1997). On the number and size of nations. The Quarterly Journal of Economics, 112(4), 1027-1056.

Alesina, A., \& Tabellini, G. (1989). External debt, capital flight and political risk. Journal of international Economics, 27(3-4), 199-220.

Amin, S. (2019). The endless nexus between ethnic diversity, social exclusion and institutional quality of Pakistan. International Journal of Sociology and Social Policy, 39(3/4), 182-200.

Amin, S., \& Ahmad, N. (2018). Ethnic Diversity, Social Exclusion and Economic Determinants of Crimes: A Case Study of Pakistan. Social Indicators Research, 140(1), 267-286.

Ananiev, J., Atanasov, P., Gerovska-Mitev, M., \& Shukarov, M. (2011). Beyond Transition Towards Inclusive Societies.

Appasamy, P., Guhan, S., Hema, R., Majumdar, M., \& Vaidyanathan, A. (1996). Social exclusion from a welfare rights perspective in India: International Institute for Labour Studies:.

Azam, J.-P. (2001). The redistributive state and conflicts in Africa. Journal of Peace research, 38(4), 429-444.

Baltagi, B. H., \& Kao, C. (2001). Nonstationary panels, cointegration in panels and dynamic panels: A survey Nonstationary panels, panel cointegration, and dynamic panels (pp. 7-51): Emerald Group Publishing Limited.

Bardsley, M., \& Flatley, J. (1998). The people of London: social and economic factors in health. The health of Londoners: a public health report for London eds. Bardsley, M., Barker, M. et al. London, published on behalf of the Health of Londoner's Project by King's Fund Publishing, 11-24.

Barry, B. (2002). Social exclusion, social isolation, and the distribution of income. Understanding social exclusion, 13-29.

Barth, F. (1998). Ethnic groups and boundaries: The social organization of culture difference: Waveland Press.

Bates, R. H. (2000). Ethnicity and development in Africa: A reappraisal. The American Economic Review, 90(2), 131-134.

Becker, G. S. (1962). Irrational behavior and economic theory. Journal of political economy, $70(1), 1-13$.

Benington, J., \& Geddes, M. (2013). Local Partnership and Social Exclusion in the European Union: New Forms of Local Social Governance? : Routledge.

Castles, S. (2000). International Migration at the Beginining of the Twenty-First Century: Global Trends and Issues. International Social Science Journal, 52(165), 269-281.

Delhey, J., \& Newton, K. (2005). Predicting cross-national levels of social trust: global pattern or Nordic exceptionalism? European Sociological Review, 21(4), 311-327.

DESA, U. (2007). World Economic Situation and Prospects, 2007. New York: UN DESA.

Dincer, O. C., \& Wang, F. (2011). Ethnic diversity and economic growth in China. Journal of Economic Policy Reform, 14(1), 1-10.

Easterly, W., \& Levine, R. (1997). Africa's growth tragedy: policies and ethnic divisions. The quarterly journal of economics, 112(4), 1203-1250.

Easterly, W., Ritzen, J., \& Woolcock, M. (2006). Social cohesion, institutions, and growth. Economics \& Politics, 18(2), 103-120. 
Ellis, L., Beaver, K. M., \& Wright, J. (2009). Handbook of crime correlates: Academic Press.

Esping-Andersen, G., Gallie, D., Hemerijck, A., \& Myles, J. (2002). Why we need a new welfare state: OUP Oxford.

Fleurbaey, M. (2002). Development, capabilities, and freedom. Studies in Comparative International Development, 37(2), 71-77.

Glaeser, E. L., \& Saks, R. E. (2006). Corruption in america. Journal of public Economics, 90(6), 1053-1072.

Hooghe, M., \& de Vroome, T. (2016). The relation between ethnic diversity and fear of crime: An analysis of police records and survey data in Belgian communities. International Journal of Intercultural Relations, 50, 66-75.

Hsiao, C. (1986). Analysis of Panel Data, Econometric Society Monograph No. 11: Cambridge: Cambridge University Press.

Jehoel-Gijsbers, G., \& Vrooman, C. (2007). Explaining social exclusion.

Karlsen, S., \& Nazroo, J. Y. (2002). Relation between racial discrimination, social class, and health among ethnic minority groups. American journal of public health, 92(4), 624631.

Keen, D. (2000). Incentives and disincentives for violence: Lynne Reinner Publishers; International Development Research Centre.

La Porta, R., Lopez-de-Silanes, F., Shleifer, A., \& Vishny, R. (1999). The quality of government. The Journal of Law, Economics, and Organization, 15(1), 222-279.

Lechman, E. (2014). Socio-Economic Exclusion as a Hindrance of Economic Development. A Comparative Study for European Countries.

Levitas, R. (2004). Let's hear it for Humpty: social exclusion, the third way and cultural capital. Cultural trends, 13(2), 41-56.

Levitas, R., Pantazis, C., Fahmy, E., Gordon, D., Lloyd, E., \& Patsios, D. (2007). The multidimensional analysis of social exclusion.

Lister, R. (1998). Citizenship on the margins: Citizenship, social work and social action. European Journal of Social Work, 1(1), 5-18.

Mason, N. W., MacGillivray, K., Steel, J. B., \& Wilson, J. B. (2003). An index of functional diversity. Journal of Vegetation Science, 14(4), 571-578.

Mathieson, J., Popay, J., Enoch, E., Escorel, S., Hernandez, M., Johnston, H., \& Rispel, L. (2008). Social Exclusion Meaning, measurement and experience and links to health inequalities. A review of literature. WHO Social Exclusion Knowledge Network Background Paper, 1, 91.

McAdam, D. (2000). Culture and social movements Culture and Politics (pp. 253-268): Springer.

Miller, H. J. (2006). Social exclusion in space and time. Paper presented at the AXHAUSEN KW Moving through nets: The Physical and Social Dimensions of Travel. Selected papers from the 10th International Conference of Travel Behaviour Research $\mathrm{H}$. Elsevier Science Publishing Company.

Nafziger, E., \& Auvinen, J. (2003). Economic Development, Inequality and War: Humanitarian Emergencies in Developing Countries: Springer.

Peace, R. (2001). Social exclusion: A concept in need of definition? Social Policy Journal of New Zealand, 17-36.

Peleah, M., \& Ivanov, A. (2013). Measuring Intersecting Inequalities Through the Social Exclusion Index: A Proposal for Europe and Central Asia. United Nations Economic Commission for Europe.

Popay, J., Escorel, S., Hernández, M., Johnston, H., Mathieson, J., \& Rispel, L. (2008). Understanding and tackling social exclusion. Final report to the WHO Commission on Social Determinants of Health, from the Social Exclusion Knowledge Network. Geneva, Switzerland: WHO.

Rawal, N. (2008). Social inclusion and exclusion: A review. Dhaulagiri Journal of Sociology and Anthropology, 2, 161-180. 
Robeyns, I. (2005). Selecting capabilities for quality of life measurement. Social Indicators Research, 74(1), 191-215.

Rosen, H. S. (2004). Public finance The encyclopedia of public choice (pp. 252-262): Springer.

Schumpeter, J. A. (2017). Theory of economic development: Routledge.

Silver, H. (2007). The process of social exclusion: the dynamics of an evolving concept.

Silver, H., \& Miller, S. M. (2003). Social exclusion. Indicators, 2(2), 5-21.

Simandan, D. (2010). On how much one can take: relocating exploitation and exclusion within the broader framework of allostatic load theory. Health \& Place, 16(6), 12911293.

Sung, H. (2014). UNESCO World Culture Report. In A. C. Michalos (Ed.), Encyclopedia of Quality of Life and Well-Being Research (pp. 6773-6774). Dordrecht: Springer Netherlands.

Sutherland, A. (1997). Fiscal crises and aggregate demand: can high public debt reverse the effects of fiscal policy? Journal of public Economics, 65(2), 147-162.

Sutherland, J. (2001). Economic development vs social exclusion: The cost of development in Brazil. Paper presented at the Latin American Studies Association Conference.

Thomas, D. A., \& Ely, R. J. (2001). A new paradigm for managing diversity. Harvard business review, 74(5), 79-94.

Thorat, S., \& Neuman, K. S. (2012). Blocked by caste: economic discrimination in modern India: Oxford University Press.

Treisman, D. (2000). Decentralization and inflation: commitment, collective action, or continuity? American Political Science Review, 94(4), 837-857.

UNDP. (2011). Beyond transition: towards inclusive societies: United Nations Development Programme, Regional Bureau for Europe and CIS ....

Unit, S. E. (2004). Mental health and social exclusion: social exclusion unit report summary: Crown.

Uslaner, E. M. (2012). Segregation and mistrust: Diversity, isolation, and social cohesion: Cambridge University Press.

Van den Berghe, P. L. (1994). The quest for the other: ethnic tourism in San Cristóbal, Mexico: University of Washington Press.

Väyrynen, R. (2003). Regionalism: old and new. International Studies Review, 5(1), 25-51.

Vigoda, E. (2002). Administrative agents of democracy? A structural equation modeling of the relationship between public-sector performance and citizenship involvement. Journal of Public Administration Research and Theory, 12(2), 241-272.

Vrooman, J. C., \& Hoff, S. J. (2013). The disadvantaged among the Dutch: A survey approach to the multidimensional measurement of social exclusion. Social Indicators Research, 113(3), 1261-1287.

Walsh, T. (2006). A right to inclusion? Homelessness, human rights and social exclusion. Australian Journal of Human Rights, 12(1), 185-204.

Wolff, J., \& De-Shalit, A. (2007). Disadvantage: Oxford University Press on Demand.

Young, I. M. (2002). Lived body vs gender: Reflections on social structure and subjectivity. Ratio, 15(4), 410-428.

\section{Biographical Notes}

Mr. Saqib Amin is a PhD Scholar at the National College of Business Administration \& Economics, Pakistan. 


\section{Appendix}

\section{List of Countries:}

\begin{tabular}{|c|c|c|c|c|}
\hline Andorra & Ireland & Qatar & Samoa & Eritrea \\
\hline Antigua and Barbuda & Israel & San Marino & Sao and Principe & Ethiopia \\
\hline Australia & Italy & Saudi Arabia & Serbia and Monten. & Gambia, The \\
\hline Austria & Japan & Seychelles & Solomon Islands & Guinea \\
\hline Bahamas, The & Kuwait & Singapore & South Africa & Guinea-Bissau \\
\hline Bahrain & Latvia & Slovak Rep. & Sri Lanka & Haiti \\
\hline Barbados & Liechtenstein & Slovenia & St. Lucia & Zimbabwe \\
\hline Belgium & Lithuania & Spain & Sudan & Senegal \\
\hline Brunei Darussalam & Luxembourg & Sweden & Afghanistan & Sierra Leone \\
\hline Canada & Malta & HK, China & Benin & Somalia \\
\hline Chile & Monaco & Hungary & Burkina Faso & Tanzania \\
\hline Cyprus & Netherlands & Iceland & Burundi & Togo \\
\hline Czech Republic & New Caledonia & Switzerland & Central African Rep. & Uganda \\
\hline Denmark & New Zealand & Trinidad \& Tob. & Chad & Rwanda \\
\hline Estonia & Norway & UAE & Comoros & Liberia \\
\hline Finland & Oman & UK & Congo, Dem. Rep. & Madagascar \\
\hline France & Palau & United States & Nepal & Malawi \\
\hline Germany & Poland & Uruguay & Niger & Mali \\
\hline Greece & Portugal & Fiji & Kiribati & Mozambique \\
\hline Albania & Cameroon & Gabon & Kyrgyz Republic & Pakistan \\
\hline Algeria & Cape Verde & Georgia & Lao PDR & Panama \\
\hline Angola & China & Ghana & Lebanon & Pap. New Guinea \\
\hline Argentina & Colombia & Grenada & Lesotho & \\
\hline Armenia & Congo, Rep. & Guatemala & Libya & \\
\hline Azerbaijan & Costa Rica & Guyana & Macedonia, FYR & \\
\hline Bangladesh & Cote d'Ivoire & Honduras & Malaysia & \\
\hline Belarus & Croatia & India & Maldives & \\
\hline Belize & Cuba & Indonesia & Marshall Islands & \\
\hline Bhutan & Djibouti & Iran, Rep. & Mauritania & \\
\hline Bolivia & Dominica & Iraq & Mauritius & \\
\hline Bosnia and Herz & Dominican Rep. & Jamaica & Mexico & \\
\hline Botswana & Ecuador & Jordan & Micronesia, Fed. Sts. & \\
\hline Brazil & Egypt, Arab Rep. & Kazakhstan & Moldova & \\
\hline Bulgaria & El Salvador & Kenya & Mongolia & \\
\hline Cambodia & Equatorial Guinea & Timor-Leste & Turkey & \\
\hline Suriname & Taiwan, China & Tonga & Turkmenistan & \\
\hline Swaziland & Tajikistan & Tunisia & Ukraine & \\
\hline Syrian Arab Republic & Thailand & Vanuatu & Uzbekistan & \\
\hline Venezuela, RB & Yemen, Rep. & Morocco & Paraguay & \\
\hline Vietnam & Zambia & Namibia & Peru & \\
\hline Nicaragua & Philippines & Russia & & \\
\hline
\end{tabular}




\section{Variables Description and Data Source}

\begin{tabular}{|c|c|c|}
\hline Indicators and Description & Definition & Data Source \\
\hline Analysis for Ethnic Diversity & $\begin{array}{l}\% \text { of population with ethnic groups (out of total } \\
\text { population) and used formulation of Alesina et al. } \\
\text { (2003) for its calculations. }\end{array}$ & $\begin{array}{l}\text { Database of the Cline Center } \\
\text { for Democracy }\end{array}$ \\
\hline Analysis for Religious Diversity & $\begin{array}{l}\% \text { of population with religious groups (out of total } \\
\text { population) and used formulation of Alesina et al. } \\
\text { (2003) for its calculations. }\end{array}$ & $\begin{array}{l}\text { Database of the Cline Center } \\
\text { for Democracy }\end{array}$ \\
\hline \multicolumn{3}{|l|}{ Economic Exclusion } \\
\hline Unemployment rate & Unemployment, total ( $\%$ of total labor force) & World Bank Indicator \\
\hline Gini Coefficient & GINI index (World Bank estimate) & World Bank Indicator \\
\hline Depth food deficit & Depth food deficit (kilocalories per person per day) & World Bank Indicator \\
\hline Poverty (head count ratio) & headcount ratio at $\$ 1.90$ a day (2011 PPP) (\% & UNICEF \\
\hline Poverty (Gap Ratio) & Poverty gap at $\$ 1.90$ a day (2011 PPP) (\%) & UNICEF \\
\hline Corruption Ratio & International Corruption perception index & World Bank Indicator \\
\hline Intentional homicides & Intentional homicides (per 100,000 people) & World Bank Indicator \\
\hline Out of pocket health expenditure & $\begin{array}{l}\text { Out-of-pocket expenditure (\% of current health } \\
\text { expenditure) }\end{array}$ & International Monetary Fund \\
\hline \multicolumn{3}{|l|}{ Public Service Exclusion } \\
\hline$\overline{\text { Lack of drinking water }}$ & $\begin{array}{l}\text { People using at least basic drinking water services ( } \% \\
\text { of population) }\end{array}$ & UNICEF \\
\hline Out of school children & Children out of school (\% of primary school age) & World Bank Indicator \\
\hline Life time risk of maternal death & Lifetime risk of maternal death $(\%)$ & UNICEF \\
\hline Low birth weight babies & Low-birthweight babies (\% of births) & World Bank Indicator \\
\hline Maternal mortality ratio & $\begin{array}{l}\text { Maternal mortality ratio (modeled estimate, per } \\
100,000 \text { live births) }\end{array}$ & World Bank Indicator \\
\hline Mortality rate under 5 years & Mortality rate, under-5 (per 1000 live births) & World Bank Indicator \\
\hline Number of under 5 deaths & Number of children dying before reaching age five. & World Bank Indicator \\
\hline Prevalence of undernourishment & Prevalence of undernourishment (\% of population) & World Bank Indicator \\
\hline Prevalence of underweight & $\begin{array}{l}\text { Prevalence of underweight, weight for age ( } \% \text { of } \\
\text { children under } 5 \text { ) }\end{array}$ & UNICEF \\
\hline Children living with HIV & Children (0-4) living with HIV & UNICEF \\
\hline Contraceptive prevalence & $\begin{array}{l}\text { Contraceptive prevalence, any methods ( } \% \text { of women } \\
\text { ages } 15-49)\end{array}$ & UNICEF \\
\hline Incidence of HIV & HIV infections (populations ages $15-49$ ) & UNICEF \\
\hline Incidence of tuberculosis & Incidence of tuberculosis (per 100,000 population) & UNICEF \\
\hline \multicolumn{3}{|l|}{ Civic \& Public Participation } \\
\hline \multicolumn{3}{|l|}{ Exclusion } \\
\hline Vulnerable employment & Vulnerable employment, total ( $\%$ of total employ.) & World Bank Indicator \\
\hline Gender inequality & 1 minus the index of gender equality & Indices of social development \\
\hline Lack of civic activism & 1 minus the index of civic activism & Indices of social development \\
\hline Lack of intergroup cohesion & 1 minus the index of intergroup cohesion & Indices of social development \\
\hline Less of club \& membership & 1 minus the index of club \& membership & Indices of social development \\
\hline Less intergroup safety $\&$ trust & 1 minus the index of intergroup safety $\&$ trust & Indices of social development \\
\hline Territory of asylum & Refugee population by country or territory of asylum & World Bank Indicator \\
\hline International migrants & $\%$ of people other than they lived. & World Bank Indicator \\
\hline \multicolumn{3}{|l|}{ Control variables } \\
\hline$\overline{\mathrm{GDPPC}}$ & GDP per capita (Current US \$) & World Bank Indicator \\
\hline Urbanization & Urban population ( $\%$ of total) & World Bank Indicator \\
\hline Education expenditure & Expenditure on Education (\% of GDP) & World Bank Indicator \\
\hline Pop. Density & Population density (people per sq. km of land area) & World Bank Indicator \\
\hline Health expenditure & Expenditure on Health (\% of GDP) & World Bank Indicator \\
\hline Literacy rate & Literacy rate, youth total (\% of people ages $15-24)$ & World Bank Indicator \\
\hline Institutional Quality & Absolute legal institutional quality (simple aveg.) & Kuncic, A. (2014). \\
\hline
\end{tabular}

